\title{
LETTERS
}

\section{Propofol sedation and colonoscopy: a perspective from endoscopists}

We read with interest Dr. Grocott's letter ${ }^{1}$ promoting the use of propofol sedation during colonoscopy in response to our publication. ${ }^{2}$ Dr. Grocott expressed concerns about our commentary and felt it gave a "distorted picture of clinical reality." The letter called many of our statements "unsubstantiated, speculative and even contradictory." We appreciate the opportunity to address these concerns.

We would like to offer the perspective of endoscopists. In Canada and worldwide, there is marked variation in colonoscopic technique among individual endoscopists, leading to disparate outcomes in cecal intubation, adenoma detection, postcolonoscopy colorectal cancer, patient satisfaction, and the type and amount of sedation used. Training in modern colonoscopic techniques can improve the quality of colonoscopies while lowering the amount of conscious sedation required. To address this gap, the Canadian Association of Gastroenterology has implemented programs to improve colonoscopy teaching to trainees and to improve the skills of colonoscopists in practice.

Dr. Grocott's assertion that propofol improves "efficiency" is debatable, given that quicker patient recovery does not necessarily lead to improved efficiency. A recent Canadian study showed that the use of propofol during colonoscopy resulted in prolongation of total procedure time by three minutes per case compared with traditional sedation. ${ }^{3}$ Over the course of a full day, this lost time could actually impede efficiency. Moreover, colonoscopists who use optimal techniques require minimal or no sedation of patients, leading to faster patient recovery and negating the putative benefits of propofol.

While the published literature does not clearly show a relation between level of sedation and the cecal intubation rate or the adenoma detection rate, it does not include examinations performed using modern colonoscopic techniques, such as patient repositioning and water immersion. These techniques can both improve patient comfort and increase adenoma detection. In our commentary, we highlight the fact that deep sedation using propofol discourages the use of these evidence-based best practices and thus impairs efficiency in terms of important clinical outcomes.

While Dr. Grocott states that "all of the evidence needs to be thoroughly examined in a more balanced fashion," the key points of our commentary were not addressed. The added costs of anesthesia personnel and equipment used with propofol were not discussed. It is not surprising that the small meta-analysis Dr. Grocott referenced failed to show an effect of propofol on uncommon but serious adverse events. ${ }^{4}$ In contrast, a study of more than three million colonoscopies showed an increased risk of perforation, hemorrhage, stroke and complications secondary to anesthesia in patients who had colonoscopy with anesthesia services. ${ }^{5}$ The possibility of more adverse events mandates caution and supports a change in practice. This situation is similar to the change in practice that occurred when sodium phosphate bowel preparations were associated with serious adverse events. Dr. Grocott's letter provided no rational for the continued use of propofol sedation in this context.
We strongly disagree that our commentary lacks "patient centricity." Indeed, our primary focus is to improve the quality of patient care by encouraging a frank and transparent discussion about this issue. At a minimum, patients must be fully informed about the risks and benefits of propofol sedation and be allowed to choose for themselves.

There are many perspectives and competing interests regarding sedation choice for colonoscopy. By keeping our focus on procedural efficacy, cost-effectiveness and patient safety, we maintain that for routine colonoscopy, deep sedation with propofol is wasteful and unnecessary.

\section{David E. Pace MB Admin MD}

General surgeon, Memorial University of Newfoundland, St. John's, NL

\section{Mark R. Borgaonkar MD MSC}

Gastroenterologist, Memorial University

of Newfoundland, St. John's, NL

Cite as: CMAJ 2018 November 19;190:

E1368. doi: 10.1503/cmaj.70575

\section{References}

1. Grocott HP. Propofol sedation improves efficiency and optimizes patient satisfaction during colonoscopy [letter]. CMAJ 2018;190:E751.

2. Pace D, Borgaonkar M. Deep sedation for colonoscopy is wasteful and unnecessary. CMAJ 2018;190:E153-4.

3. Thornley P, Al Beshir M, Gregor J, et al. Efficiency and patient experience with Propofol vs conventional sedation: a prospective study. World J Gastrointest Endosc 2016;8:232-8.

4. Singh H, Poluha W, Cheung M, et al. Propofol for sedation during colonoscopy. Cochrane Database Syst Rev 2008;(4):CD006268.

5. Wernli KJ, Brenner AT, Rutter CM, et al. Risks associated with anesthesia services during colonoscopy. Gastroenterology 2016;150:888-94.

Competing interests: None declared. 